\title{
Wie fokussiert man im Ägyptischen ein direktes Objekt? ${ }^{1}$
}

\section{Der Begriff des Fokus}

Der grammatische Terminus des Fokus bezeichnet die Tatsache, dass ein Satzglied als neue, noch unbekannte Information herausgestellt und so vom Rest des Satzes abgegrenzt wird, der demgegenüber als bekanntes oder als selbstverständlich zu erschließendes Wissen gilt ${ }^{2}$. In vielen, vielleicht allen Sprachen der Welt kann man eine Fokusfunktion dadurch zum Ausdruck bringen, dass man das zu fokussierende Satzglied zum Prädikat eines Nominalsatzes macht: „X ist es, der das und das getan hat" oder ,wer das und das getan hat, das ist $\mathrm{X}$ “ ( $\mathrm{X}$ fokussiert). Fragewörter wie „wer", „was", „wo" etc. sind grundsätzlich fokussiert; dasselbe gilt für die ihnen entsprechenden Elemente in einer folgenden Antwort. Im Deutschen ist die explizite Fokusmarkierung eines Frageworts im Prinzip immer möglich (,wer ist es, der . . ??", ,wo ist es, dass ...?" etc.), doch gilt sie nicht als notwendig und wird in der Praxis nur selten angewendet.

Mein Dank gilt Wolfgang Schenkel (Tübingen), der eine Version dieses Textes kommentiert hat. Dankbar erwähnt seien auch die im Internet zugänglichen Ressourcen des Thesaurus Linguae Aegyptiae (http:// aaew.bbaw.de/tla), die ich mit Gewinn konsultiert habe.

${ }^{2}$ Nach einer klassischen Definition von Jackendoff (1972: 16) ist der Fokus, the information in the sentence that is assumed by the speaker not to be shared by him and the hearer".

Vom Begriff des Fokus scharf zu unterscheiden ist der Begriff des Topic, der nahezu das Gegenteil bedeutet und doch in älteren Arbeiten unter dem unklaren Begriff der „Betonung" oft mit dem Fokus zusammengeworfen wurde. Das Topic hebt ein Element als schon bekanntes Wissen hervor und wird im Ägyptischen typischerweise mit $j r$ eingeleitet. Ein topikalisiertes direktes Objekt liegt z. B. vor in jr grt hnw nb $n$ J Jnpw ntj $m-c=k$, jmi $n=f$ sw (pHeqanakhte II $3 \dot{4}=$ Allen 2002: Tf. 30), ,was alle Habe des Jnpw betrifft, die bei dir ist (sie wird als dem Adressaten bekannt vorausgesetzt), so gib sie ihm zurück!“"
In Sprachen, die häufiger von solchen Markierungen Gebrauch machen - und dazu gehört auch das Ägyptische - kann es vorkommen, dass sich in einem Grammatikalisierungsprozess spezialisierte Fokuskonstruktionen ausbilden, die dann nicht mehr mit den gewöhnlichen Nominalsätzen identisch sind. Für solche von Nominalsätzen differenzierten Fokuskonstruktionen ist in der Ägyptologie der Begriff der Cleft-Sentence gebräuchlich. Im Laufe der ägyptischen Sprachgeschichte ist eine Ausdifferenzierung von Cleft-Sentences aus ursprünglichen Nominalsätzen mehrere Male zu beobachten.

\section{Subjektfokussierung}

Die übliche Methode zur Fokussierung eines Subjekts im Älteren Ägyptisch ist bekanntlich die Konstruktion jn + Subjekt + aktives Partizip. Diese mag in vorhistorischer Zeit aus einer Art Nominalsatz hervorgegangen sein, ist aber in dieser Form mit keiner allgemein als Nominalsatz funktionierenden Konstruktion mehr identisch. Die jn-Konstruktion kann keine anderen Satzglieder als das Subjekt (Agens) fokussieren.

Als weniger bekannte Nebenform hierzu gibt es noch eine Cleft-Sentence ohne jn, die also nur aus Subjekt + aktivem Partizip besteht (hierüber siehe Vernus 1987: 175-181). Auch wenn ein Fragewort ptr oder zy als (scheinbares) Subjekt verwendet wird, steht nie $j n$. In diesen Fällen ist allerdings nicht ganz leicht zu entscheiden, ob eine subjektfokussierende Cleft-Sentence ohne jn oder eher ein Adjektivsatz vorliegt (vgl. Vernus 2006: 153-155):

1. (ein Zylinder hat die und die Ausmaße,) ptr h33.t $r=f m \check{s} s r$ (Rhind $43=$ Peet 1923: Tf. N)

„Wieviel an Getreide geht hinein?"

2. ptr rh mj $R^{\top}$ (pHearst $11,12=$ Grapow 1958: 440)

„Wer weiß (Bescheid) wie Re?“ 
3. $z y z p \cdot t(j)=f(j)($ PT 438c)

„Wer wird übrigbleiben?"

Das Fragewort $m$,,wer; was“ steht in Subjektsfunktion dagegen immer mit $j n$ und wird mit diesem bekanntlich schon früh univerbiert ( $n m$, koptisch NIm).

\section{Objektfokussierung: Einführung}

Neben der Cleft-Sentence zur Subjektfokussierung verfügt das Ägyptische auch über ein Mittel zur Fokussierung von Adverbialien: Dieses sind die wohlbekannten Zweiten Tempora, auf die wir weiter unten noch zu sprechen kommen werden.

Nachdem wir also wissen, dass das Ägyptische über spezielle Mittel zur Fokussierung von Subjekten und von Adverbialien verfügt, drängt sich die Frage auf, ob es im Ägyptischen denn nicht auch eine Konstruktion zur Fokussierung eines direkten Objekts gibt. Was ist die ägyptische Entsprechung zu „es ist ein Haus, was ich sehe"/,ich sehe (Fokus:) ein Haus"?

Man könnte zunächst erwarten, dass eine Objektfokussierung sich umschreiben ließe, indem man die $j n$-Konstruktion statt mit einem aktiven Partizip mit einem passiven Partizip oder einer Relativform verwendete. Damit würde die prototypische Konstruktion für die Subjektfokussierung auf einem Umweg auch zur Fokussierung des (logischen) Objekts eingesetzt werden * ,ein Haus ist es, was (von mir) gesehen wird“ $=*$,ich sehe (Fokus:) ein Haus“. Von dieser theoretischen Möglichkeit wird, wie hervorzuheben ist, im Ägyptischen kein Gebrauch gemacht (siehe Edel 1955/64: § 954; Gardiner 1957: \373; Doret 1991: 91; Vernus 1991: 338; die beiden Letzteren mit Hinweisen auch auf potentielle, aber sehr unsichere Gegenbeispiele).

\section{Relativform (passives Partizip) + Objekt}

Ich möchte im Folgenden darstellen, dass das Ägyptische auch für die Objektfokussierung eine spezialisierte Cleft-Konstruktion besitzt, und zwar den Satztyp Relativform + fokussiertes Objekt.
Obwohl dieser Satztyp in den Texten gut belegt ist und Beispiele für ihn gelegentlich zitiert wurden (Sethe 1916: 32f.; Westendorf 1962: $\int 401$; Borghouts 1986: 65), hat man ihn bisher nicht als Konstruktion eigener Art wahrgenommen.

Für das älteste Ägyptisch ist ein Satztyp des zweigliedrigen Nominalsatzes vorauszusetzen. Er ist jedoch schon im Alten Reich und erst recht im Mittleren Reich nicht mehr produktiv, sondern auf bestimmte Relikte beschränkt wie zum Beispiel die Formel $X r n=f$, sein Name ist $X^{\text {‘ }}$ (eine Übersicht über diese Relikte gibt Gilula 1976: 169-171). Auch die hier besprochene objektfokussierende Konstruktion wird aus einem zweigliedrigen Nominalsatz hervorgegangen sein, muss aber innerhalb des Sprachsystems des historischen Älteren Ägyptisch ebenso als eigenständiger Satztyp aufgefasst werden wie $j n$ + Nomen + aktives Partizip. Wir wollen die jnKonstruktion als subjektfokussierende Cleft-Sentence, die Konstruktion Relativform + Objekt aber als objektfokussierende Cleft-Sentence bezeichnen. Einige Beispiele für die objektfokussierende CleftSentence sind folgende:

4. jw šms. $n=(j) n z w-b j t{ }^{\top} 3-h p r-n-R^{\complement} m 3^{\complement}-h r w$, jni.t. $n=(j)$

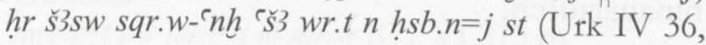
12-14)

„Ich folgte dem seligen König Thutmosis II (sc. auf einem Feldzug). Ich erbeutete im Beduinenland (Fokus:) sehr viele Gefangene, die ich gar nicht zählen kann."

Dass hier Beute gemacht wurde, wird als selbstverständlich vorausgesetzt, zumal auch auf allen zuvor erzählten Feldzügen Beute gemacht wurde. Die als neu fokussierte Information ist dagegen die genaue Natur der Beute, also das (logische) direkte Objekt. Sozusagen historisierend könnte man übersetzen *,was ich im Beduinenland erbeutete, waren sehr viele Gefangene", aber in Wirklichkeit liegt synchron keine Nominalsatzstruktur mehr vor.

5. (die Priester sollen für Hapidjefa den Totenkult vollziehen,) rdi.t.n=f $n=s n h r=s j t j-m h$ h $h$ q. $t n$ r h.t nb.t n.t pr-d.t (Siut I $279=$ Montet 1930-35: 56; ähnlich im selben Text noch öfter)

„Er hat ihnen dafür garantiert (Fokus:) einen Scheffel Breitgerste von jedem Feld des Stiftungsgutes.“ 
6. šzp.t rmt.w qrs=sn ḩ $3=s m t h \underline{h} 3=s m$ hnq.t (PT 474 $\mathrm{b}, \mathrm{c})$

„Die Menschen empfangen, wenn sie bestattet worden sind, (Fokus:) ihre tausend Brot und ihre tausend Bier."

7. wnm.t.n $=k j$ r.t (PT $192 \mathrm{~b})$

„Du hast (Fokus:) ein Auge gegessen."

8. mrr.t $k 3=f$ wnm $t($ ?) $h r$ h h3w.t $n b-r-d r$ (FoissyAufrère et al. 1985: 33,11; 18. Dyn.)

„Sein Ka möchte (Fokus:) vom Altar des Allherrn Brot(?) essen."

9. swn.t (..) Sbk-nht hhe (..) Kbsj (. .): rdid.t.n (..) Sbk-nht $n$ (...) Kbsj nbw dbn 60 (Stèle Juridique 13f. = Lacau 1949: 24)

„Handel zwischen (...) Sobeknacht und (...) Kebsi: (...) Sobeknacht gab dem (...) Kebsi (Fokus:) 60 Deben Gold.“

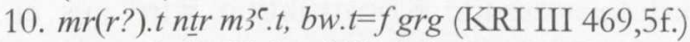

„Gott liebt (Fokus:) Wahrheit, sein Abscheu ist Lüge."

11. jrr.t j3w $n$ rmt. $w$ bjn $m$ h.t $n b . t$ (pPrisse $5,2=$ Žába 1956: 16)

„Das Alter tut den Menschen (Fokus:) Schlechtes in jeder Hinsicht."

Der letzte Beleg ist weniger klar, weil auch ein Pseudoverbalsatz mit bjn als stativischem Prädikat vorliegen könnte: ,was das Alter den Menschen tut, ist schlecht in jeder Hinsicht". Funktional würde beides praktisch auf das Gleiche hinauslaufen.

Ziemlich häufig kommt die objektfokussierende Cleft-Sentence in der Einleitung einer Rede vor, wenn die Tatsache, dass gesprochen wird, selbstverständlich ist (z. B. in einer Antwortsituation), aber der Inhalt der Rede als neue Information herausgestellt werden soll:

12. dd.t.n $n=j b 3=j$ : „.. “ (Lebensmüder 30f. $=\mathrm{Bar}-$ ta 1969: Tf. 2)

„Mein Ba sagte mir (Fokus:) ,...““

13. dd.t.n Jpw-wr wšb $=f n h m n n b-r-d r$ : , „. “ (Admonitions 15, 13 = Helck 1995: 66)

„Ipuwer sagte, indem er der Majestät des Allherrn antwortete (Fokus:) ,... . “

14. dd.t.n $z 3 b\langle r\rangle-N h n$ Sbk-m-s3=f: „... (RandallMaciver \& Woolley 1911: 182; 18. Dyn.)

„Der Richter und Sprecher-von-Hierakonpolis Sebekemsaf sagte (Fokus:) ,...“

15. dd.t ntr pn $\mathrm{C}$ hft spr=fr $j$ jmj.w=s: ,... "(Piankoff 1944: Tf. 11,1)

„Wenn der große Gott zu ihren (= der Höhle) Einwohnern gelangt, dann sagt er (Fokus:) ,... ‘“

16. $\underline{d}$ d.jn $h m=f \quad n=s n:$ nttin $j h$, d d.t. $n=s n: t w=n\langle n\rangle$ p3 wr $n$ Ht 3 (KRI II 110)
„Da sprach Seine Majestät zu ihnen: «Was seid ihr?» Sie antworteten (Fokus:) «Wir sind vom Fürsten von Chatti.»"

17. dd.t.n rwd $w$ Hcy: ,. . “ “ (Mes N23 = KRI III 429) „Der Inspektor H'y sagte (Fokus:) ,. . “““

Wenn auf die Bezeichnung des Agens verzichtet werden soll, wird die Abfolge Relativform + Objeket durch die Abfolge passives Partizip + Passivsubjekt ( = semantisches Objekt) ersetzt:

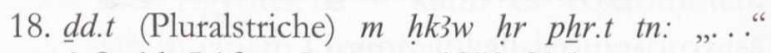
(pSmith 5,1f. $=$ Grapow 1958: 314)

„Als Zauber über diesem Medikament ist zu sprechen (Fokus:) ,...““

Wie verbreitet im Ägyptischen derartige Konstruktionen bei der Redeeinführung waren, ist auch daran abzulesen, dass das koptische Verb $\boldsymbol{\Pi \in \boldsymbol { x }} \mathbf{\alpha}$, ,er sagte“ etymologisch auf eine Relativform zurückgeht (*, was er sagte, war: ,...“).

\section{Objekt + Relativform}

Wenn das fokussierte Objekt ein Fragepronomen ist, so wird die Reihenfolge obligatorisch invertiert und es entsteht die Abfolge fokussiertes Objekt + Relativform. Während bekanntlich im ägyptischen Verbalsatz Fragewörter nicht grundsätzlich wie in europäischen Sprachen am Satzanfang stehen müssen, nimmt im Nominalsatz beliebigen Typs das Fragewort in aller Regel die erste Stelle ein Junge 1981: 435-438); diese Regel wirkt offensichtlich auch in der objektfokussierenden Cleft-Sentence:

19. jšst tr mn.t.n ntr ${ }^{3}$ (CT III 3 e)

„Woran ist denn der große Gott erkrankt?"

20. (j)h jri.t $=s r=t n$ p 3 msdi $s(j)$ (pHeqanachte I vs. 15 = Allen 2002: Tf. 28)

„Was hat sie euch getan, oh du, der du sie hasst?"“

Besonders zahlreich sind Beispiele mit ptr/ $p w$-tr, die an der Oberfläche genauso aussehen, aber möglicherweise (siehe oben) als Adjektivsätze zu verstehen und dann nicht hierher gehörig wären:

21. ptr ssn.t $t \underline{t}$ (pKahMed 1,6 = Grapow 1958: 458) "Was riechst du?"

22. $p w$-tr jri.t $=n \quad r=s$ (Admonitions 3, $13=$ Helck 1995: 14)

„Was können wir dagegen tun?" 
23. ptr $\underline{d}$ d.t $n=j n b=j$ (Sinuhe B $261=$ Koch 1990: 75)

„Was sagt mir mein Herr?"

24. ptr jri.t $=j n=\underline{t} n$ (Westcar 11,6f. = Blackman 1988: 14)

„Was kann ich für euch tun?“

25. ptr $m 3 . t . n=k$ (Var.: $m 3 . n=k$ ) $j m$ (Tb 125 Schlussrede $=$ Naville 1886, II: 324)

"Was hast du dort gesehen?"

26. ptr jri.t. $n=k$ jri.tw $r=k$ (Sinuhe B $183=$ Koch 1990: 60)

„Was hast du getan, so dass man gegen dich vorgehen müsste?"

Im folgenden Beispiel wird die Relativform durch eine passive Relativphrase ersetzt, so dass das Agens implizit bleibt wie in Bsp. (18):

27. $p w$-tr $n t j-t w$ r jri.t (Admonitions 4, 6f. $=$ Helck 1995: 19)

„Was soll man tun?"

Gelegentlich und besonders im Alten Reich ist mit einer Initialstellung des fokussierten Objekts auch außerhalb der Bedingung zu rechnen, dass dieses ein Fragewort ist. Kandidaten hierfür sind etwa folgende:

28. m35.t mrr.t ntr (Urk I 195,7)

„Gott liebt (Fokus:) Wahrheit.“

29. $m 3$ 3 $t$ dd.t.n NN (PT $2290 \mathrm{~b}=$ Faulkner 1969: 88)

"NN hat (Fokus:) die Wahrheit gesagt."

30. $n d s$ s wm.t.n Stš jm=s (PT 61a und 88c)

„Seth hat (Fokus:) (nur) wenig davon gegessen."

31. htp.t m33.t=k (PT $34 \mathrm{c})$

„Was du siehst, ist Opferspeise/du siehst (Fokus:) Opferspeise.“

Von spezieller Natur ist der folgende Fall, in dem das fokussierte Objekt negiert ist:

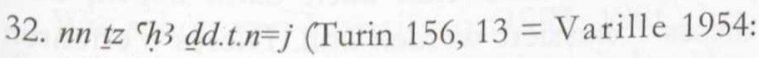
132; 18. Dyn.)

„(Man fragte mich um Rat, weil ich Gutes sagte,) es waren keine Streitworte, was ich sagte."

\section{Objekt $+p w+$ Relativform}

Es kann aber auch der eigentlich produktive Nominalsatztyp des Mittelägyptischen, nämlich der dreigliedrige Nominalsatz A pw B, zur Objektfokussierung eingesetzt werden (vgl. Edel 1955/64: \968, der von „Umformungen eines Aussagesatzes mit betontem Objekt" spricht). Dieser Fall ist in unserem Zusammenhang nicht besonders interessant, weil der Nominalsatz nicht nur speziell Objekte, sondern prinzipiell alle nominalen Elemente fokussieren kann. Im Regelfall steht dabei das fokussierte Element links von $p w$. Einige Beispiele:

\section{3. jšst $p w$ jr $=$ fjri.t $t=k n=j m$ jsw jr(j) (CT VI $250 \mathrm{~g}$ )}

„Was tust du mir denn als Gegenleistung dafür?"

(Aus dem Sargtextspruch 629, der eine Reihe weiterer Fragen entweder nach dem Subjekt (in Form der $j n$-Konstruktion) oder nach einer Adverbiale (in Form eines Zweiten Tempus) enthält.)

34. jšst pw m3.t.n=k jm (CT VII $212 \mathrm{~h}$ )

„Was hast du dort gesehen?"

35. jsf.t $p w \operatorname{jrr}(. t)=s n$ (Admonitions 5, $4=$ Helck 1995: 23)

„Sie tun (Fokus:) Unrecht.“

36. (Nemtinacht droht dem Bauern, der den Vermögensverwalter Rensi einschalten will:) jnk $p w$ $m d w n=k, m r-p r p w \quad s h 3 y=k$ (Bauer B1 51f. $=$ Parkinson 1991: 12f.)

„(Fokus:) (Einer wie) ich spricht mit dir, und du erwähnst (Fokus:) den Vermögensverwalter?"“

37. $r n$ pw $n f r$ sh3.w $=\underline{t} n$ (Klebs 1922: 22, Z. 6; weitere Belege für diese Formel bei Lacau 1961: 223f.)

„Ihr sollt euch (Fokus:) an einen guten Namen erinnern."

38. $m r r . t=\underline{t} p w$ jrr.t $t=\underline{t} m$ ntr.w (CT VI 273 b)

„Du (= Sachmet) tust (sogar) unter den Göttern

(Fokus:) (nur das) was du willst."

Auch hier kann die Relativform wieder durch ein passives Partizip ersetzt werden, wenn das Agens nicht bezeichnet werden soll:

39. jšst pw jry.t (Admonitions 5, $10=$ Helck 1995: 25)

„Was kann man tun?"

40. jw $m f k 3 . t m \underline{d} w r n h h, j n m p w w h 3 r$ tr $p n$ (Sinai 90,9f. $=$ Gardiner \& Peet 1952/5: Tf. 25a)

„Es gibt immer Türkis im Berg, (aber) zu dieser Jahreszeit sucht man (Fokus:) die (richtige) Farbe."

\section{Relativform $+p w+$ Objekt}

Nur ganz sporadisch sind Beispiele anzutreffen, in denen man mit einer umgekehrten Reihenfolge rechnen muss. So kann in den folgenden Fällen die Fokusposition wohl nur rechts von $p w$ liegen: 
41. dd.t. $n=s n$ pw ,..." (pBerlin 10003A II,17 = Sethe 1928: 96; 12. Dyn.)

„Sie sagten (Fokus:) ,... ."،

42. $\underline{\text { dd.t. } n=f p w ~ \# . . . ~(S t e ̀ l e ~ j u r i d i q u e ~} 19=\mathrm{Lacau}$ 1949: 35)

„Er sagte (Fokus:) ,. . ، “

Hier mag die Länge des fokussierten Objekts, das aus der ganzen Redewiedergabe besteht, eine Rolle für die Umstellung gespielt haben. Ferner könnte eine Kontamination mit der ähnlichen Konstruktion ohne $p w$ vorliegen, die wir oben besprochen haben.

\section{Neuägyptische Cleft-Sentence}

Im Neuägyptischen kommt ein neuer Typus der Cleft-Sentence mit der Struktur Nomen + definiter Artikel + Relativphrase auf. Er basiert auf dem im Neuägyptischen wieder möglich gewordenen zweigliedrigen Nominalsatz (Depuydt 1994: 65-71), beginnt sich aber bald durch Grammatikalisierung vom Nominalsatz wegzuentwickeln und ist spätestens im Koptischen auch wieder klar von ihm zu unterscheiden (einschlägig hierzu Polotsky 1962).

Die neuägyptische Cleft-Sentence dient zunächst der Fokussierung beliebiger nominaler Konstituenten mit Ausnahme des Subjekts; sie verdrängt also insbesondere die (ältere) objektfokussierende Cleft-Sentence Relativform $+\mathrm{Ob}$ jekt. Im weiteren Verlauf der Sprachgeschichte verliert sie auch noch diese Beschränkung und verdrängt auch die subjektfokussierende CleftSentence $j n+$ Subjekt + aktives Partizip. Die Verwendung der neuägyptischen $\mathrm{Cleft-Sentence} \mathrm{zur}$ Fokussierung des direkten Objekts ist gut bekannt (viele Beispiele bei Groll 1967: 67-71 und Neveu 1994: 203-205) und muss hier nicht mehr detailliert belegt werden. Wenige Beispiele genügen:

43. jh $p 3 n t j j w=n(r)$ jri $=f$ (Horus \& Seth 2,2 = LES 38)

„Was sollen wir tun?"

44. (beim Verhör:) $p 3 j r y=j{ }^{`} q 3 p 3 j \quad d d=j$ (KRI VI $805,10 f$.)

„Ich habe gesagt (Fokus:) exakt was ich getan habe."

45. $w^{\top}$ s.t $w^{c} . t j t 3 w n=n$ (KRI VI 781,6)

„Wir öffneten (Fokus:) (nur) ein einziges Grab.“
Mit passivem Partizip statt Relativform:

46. (Du schicktest eine Ladung Bleiglanz für den Pharao,) $w^{c} d b n w^{c} . t j n$ msdm.t $p 3 w$ gmy.t $j m=f$ (KRI VI 519,2f.)

„(aber) man fand darin (Fokus:) (nur) ein einziges deben Bleiglanz"

\section{Objekt mit $m$}

Zur Fokussierung von Adverbialien dienen bekanntlich die von H. J. Polotsky in dieser Funktion erkannten Zweiten Tempora. Man kann die Zweiten Tempora aber auch zur Fokussierung eines (semantischen) direkten Objekts einsetzen, indem man dieses in ein Präpositionalobjekt mit der Präposition $m$ transformiert.

Im Ägyptischen steht die Konstruktion des direkten Objekts grundsätzlich in Konkurrenz mit einer präpositionalen Anbindung. Gewisse Verben können dieselbe semantische Rolle bald durch ein direktes Objekt, bald durch ein Präpositionalobjekt zum Ausdruck bringen (vgl. Hafemann 2002: 165-180). Dabei bildet sich die Tendenz heraus, dass die Funktion eines direkten Objekts, wenn ein solches aus irgendwelchen Gründen nicht verwendet werden soll, durch ein Präpositionalobjekt speziell mit der Präposition $m$ ersetzt wird (vgl. Satzinger 1997: 147-154; Peust 2006: 239, Bsp. 202). Im Demotischen und Koptischen wird dann bekanntlich die Konstruktion des Objekts mit $m$ in bestimmten Tempora so gut wie obligatorisch (die sogenannte Stern-Jernstedt'sche Regel).

Eines der Motive für die Anbindung des Objekts mit $m$ kann nun auch darin liegen, dass dieses fokussiert werden soll. Dieses Verfahren ist prinzipiell schon bekannt. Es wurde von Silverman (1980) klar beschrieben und von mehreren anderen Forschern aufgegriffen und um Belege bereichert (Castle 1993: 107f.; Fischer-Elfert 2000: 264; Loprieno 1995: 198f.). Trotzdem wird die Häufigkeit und Bedeutung der Konstruktion insgesamt noch unterschätzt.

Von den bisher schon bekannten Belegen möchte ich hier einige besonders aussagekräftige wiederholen. Die folgenden sind bemerkenswert, weil das Verb klare second-tense-Morphologie zeigt: 
47. $j r r=k m$ hr.t $j b=k, s w r \underline{d} p w d d n=k$ (Schiffbrüchiger 20f. = Blackman 1932: 42)

„Du tust (Fokus:) (ja nur) was du willst; es ist ermüdend, dir (etwas) zu sagen (freier: dir Ratschläge zu geben).“

48. jr gr.t $n 3$ dd. $n=j n=k r-d d$ jmi $n=f j t j-m h \underline{h} 3 r n$ 3bd, $\underline{d} \underline{d}=k n=f m j t j-m h$ 0,8 $n$ 3bd (pHeqanachte I $17=$ Allen 2002: Tf. 26)

„Auch wenn ich dir gesagt habe, dass du ihm einen Sack Breitgerste pro Monat geben sollst, (so korrigiere ich das jetzt:) du sollst ihm (Fokus:) (nur) 0,8 Sack Breitgerste pro Monat geben! “

49. rh h.t $n$ rmt.w nb.t, jn $h m=k m h 3 w=j$ (Bauer B1 165f. $=$ Parkinson 1991: 26f.)

„Du Kundiger aller Menschen, solltest du (Fokus:) (ausgerechnet) meine Angelegenheit nicht verstehen?" (zu $h m=f$ als second-tense-Form siehe Peust 2006: 228)

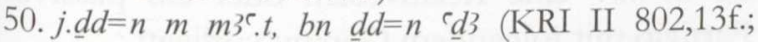
ähnlich noch mehrfach im Neuen Reich, z. B. KRI III 429,9f. und 433,9; KRI IV 318,11f.)

„Wir werden (Fokus:) die Wahrheit sagen, wir werden keine Lüge sagen."

51. $w d n$ zp-2 $n$ nb.t p.t, shtp $=t n m$ Hw.t-Hrw (Sinai 90,19f. $=$ Gardiner \& Peet 1952/5: Tf. 25a)

„Opfert, opfert der Herrin des Himmels, besänftigt (Fokus:) Hathor! ‘“

Dieser Beleg verdient vielleicht Aufmerksamkeit, weil er ein Argument für die Existenz eines Zweiten Tempus des Subjunktivs darstellen könnte, denn zur Fortsetzung des Imperativs erwarten wir den Subjunktiv (siehe Peust 2004: 338-340). Anders als etwa für den Prospektiv werden für den Subjunktiv explizite secondtense-Verwendungen bisher nicht überall anerkannt (so etwa nicht von Schenkel 2005: 235, 288; zustimmend dagegen Zeidler 1999, I: 161f.).

Wie schon von Loprieno (1995: 198f.) hervorgehoben wurde, kann auch hier wieder, ähnlich wie in den oben behandelten Konstruktionen, der Ausdruck des Agens durch eine Passivkonstruktion umgangen werden:

52. jr $\breve{s m}\{t\}$ z $3 h r w 3 . t$, gmm.tw $m$ z 2 (Admonitions 12, 13f. $=$ Helck 1995: 57)

„Wenn drei Leute sich auf den Weg machen, findet man (Fokus:) (nachher nur noch) zwei Leute (sc. weil sie einen von sich umgebracht haben)."

An neuen Belegen für das fokussierte Präpositionalobjekt möchte ich folgende in ungefährer chronologischer Folge anführen. Wie man er- kennen kann, ist die Konstruktion bis ins Neuägyptische hinein lebendig geblieben:

\section{3. $w n m=k j r=f m j$ jšst (CT III $81 \mathrm{~b}$ )}

„Was wirst du essen?"

Ein Fragewort ist ein besonders sicherer Kandidat für die Fokussierungsleistung.

54. $\underline{d i}=\underline{t}$ tn $n=j n \underline{t r} . w m$ t $m$ hnqq.t (CT III 189 a)

"Gebt mir, ihr Götter, (Fokus:) Brot und Bier.“

55. $j r r h m=k m$ mri.t $=f$ (Sinuhe B 236 und $263=$ Koch 1990: 68 und 75)

„Deine Majestät tut (Fokus:) was sie wünscht.“

Die Lesung mri.t $=f$ halte ich für sowohl paläographisch (Photographien in Gardiner 1909: Tf. 13 und 14) als auch grammatisch plausibler als die meist bevorzugte Lesung $m r r=f$.

56. jwi $n=k r m . w$ šn ${ }^{\complement} . y w, p h=k m$ 3pd.w $d d 3$ (Bauer B1 91f. = Parkinson 1991: 18)

„Die Fische werden zu dir kommen (Fokus:) indem sie $\breve{s}^{r}$ sind, du wirst erbeuten (Fokus:) fette Vögel.“

Dieser Fall zeigt Adverbfokussierung und Objektfokussierung in Parallele.

57. jh=j st $m d m j w^{c}, q d . n=j h r=s m$ jnb $n$ wmt.t (Urk IV $767,10 \mathrm{f}$.)

„Ich fing sie in einer einzigen Stadt ein, denn ich hatte um sie (Fokus:) (nicht weniger als) eine Umfassungsmauer errichtet. “

58. jmi $=k$ wnm j(3)rr.t $m$ hrw pn (...) zwr.tw $m$ jrp, wnm.tw $m$ bj.t $m$ hrw pn (Leitz 1994: 218f.)

„Du sollst an diesem Tag keine Weintrauben essen (...) man soll an diesem Tag (Fokus:) Wein trinken und (Fokus:) Honig essen."

59. jw bw gmi.tw $n=j \underline{t 3 y} m$ jri. $n=j n b$, jw j.jri $=[j] m$ 3h.w $n b n n b=j$ (KRI III 92, 11f.)

„Man fand an mir keinen Tadel in allem, was ich tat, sondern ich tat (Fokus:) all das, was meinem Herrn nützte."

60. j.jri $=\langle n\rangle \quad m$ b3k $n f r \quad m n h \quad n k$ s.t $n h h$ (KRI IV $86,5 \mathrm{f}$.)

„Wir tun (Fokus:) gute, vortreffliche Arbeit als Werk für die Ewigkeit.“

61. nttın $n 3$ nb.w n p.t $t 3 d w 3 . t$, j.jri.tw $m p 3$ j. $d d=\underline{t} n$ (KRI VI 440,4f.; ähnlich LEM 20,13f.; Černý \& Gardiner 1957: Tf. 80,20)

„Ihr seid die Herren von Himmel, Erde und Unterwelt; man tut (Fokus:) was ihr sagt."

62. jst bn j.jri.tw m p3w dd (J)tmw nb t3.wj Jwnw hn $n^{\ulcorner} p 3-$ $R^{c}-H r w-3 h t j$ (Horus \& Seth 8,5f. = LES 47)

„Soll man etwa nicht tun (Fokus:) was Atum, der Herr beider Länder, der Heliopolitaner, und ReHarachte gesagt haben?"“ 
63. j.jri=k di.t jni.tw $m n 3 n t j$ hnn(r) r-bnr $m$ sh.t, jw n3 $n t j m n 3$ mhr.w smn $(m)$ s.t $=w$ (pLeiden I 370,10f. = LRL 9)

„Du sollst (Fokus:) (nur) dasjenige (sc. Getreide), das draußen auf den Feldern verstreut ist, bringen lassen, aber das, das in den Speichern ist, soll an seinem Ort bleiben."

Mit neuägyptischer Graphie $n$ statt $m$ :

64. hr j.jri Jmn grg $n n 3$ t3.w $\underline{d} r=w$ (Wenamun 2,19f. = LES 68f.)

„Amun hat ja (Fokus:) alle Länder gegründet (gemeint: nicht nur Ägypten)."

Abschließend ist die Warnung angebracht, dass nun aber nicht jedes durch $m$ angebundene Objekt als fokussiert zu erklären ist. Für die Verwendung von $m$ kommen eine ganze Reihe von Gründen in Frage, und jeder Einzelfall muss individuell beurteilt werden.

\section{Voranstellung im Verbalsatz?}

Reintges (1998: 208) rechnet mit der Möglichkeit, dass ein Fragewort als Objekt im Ägyptischen ähnlich wie in europäischen Sprachen einfach aus einem Verbalsatz heraus nach links extrahiert werden könne. Damit hätten wir dann noch eine weitere Variante der Fokussierung, nämlich durch ein reines Wortstellungsmittel. Er bringt folgendes Beispiel:

65. $m$ hwi.n=k (Quibell et al. 1898: Tf. 33 links; 5 . Dyn.)

„Wen hast du geschlagen?"

Wir können hier aber besser den gut bestätigten, oben behandelten Satztypus Objekt + Relativform annehmen. Weitere in diesem Sinne prinzipiell ambige Fälle, in denen die Relativform morphologisch möglich, aber nicht nachweisbar ist, können sich natürlich leicht ergeben, vgl. nur noch:

66. $m$ tr $m s i n=t j 3 r$ pn (CT VI 309 j)

„Wen hat dir dieses Binsendickicht geboren?" oder evtl. auch „wen hast du (in) diesem Binsendickicht geboren?"

Man findet sporadisch Fälle, in denen bei einer mutmaßlichen neutrischen Relativform das erwartete $t$-Element fehlt und die eine Interpretation à la Reintges eher nahelegen könnten, z. B.:
67. jšst $k 3=n r$ sh.wj $p n$ (CT V $394 \mathrm{n})$

„Was planen wir für diese beiden Ehrwürdigen?"“

Da die neutrische $-t$-Endung aber auch sonst gelegentlich fehlt (Gardiner 1957: \511.4), würde ich bis auf weiteres auch hier lieber die objektfokussierende Cleft-Sentence annehmen und nicht mit der Möglichkeit einer Linksextraktion des Objekts aus dem Verbalsatz rechnen.

\section{Anruf an die Lebenden}

Nicht ganz einfach zu analysieren sind Wendungen aus den Anrufen an die Lebenden wie die folgenden, in denen auch nicht ganz klar ist, $\mathrm{ob}$ in $m r r$ eine Relativform oder ein passives Partizip mit folgendem Genitiv vorliegt:

68. $m r r . w n z w \underline{d} d . t(j)=s n$ (folgt Opferformel) (M. ElKhadragy 2002: 206 unten)

etwa: „Diejenigen, die sprechen werden „...“, die liebt der König."

69. $m r r W p\{t\}-w 3 . w t \underline{d} d . t j=f(j) \quad$ (folgt Opferformel) (Tresson 1928: 77)

etwa: „Wer sprechen wird „...“, den liebt Upuaut."

70. $m r r . y H w . t-H r w$ nb.t $m f\{w\} k 3 . t \quad \underline{d d} . t(j)=f j$ (folgt Opferformel) (Sinai $30=$ Gardiner \& Peet 1952/5: Tf. 13)

etwa: „Wer sprechen wird „...“, den liebt Hathor, die Herrin des Wadi Maghârah.“

(Weitere Belege für diesen und die folgenden Typen gibt Vernus 1976: 132.)

Grammatisch kann hier wohl nur eine subjektfokussierende Cleft-Sentence ohne $j n$ vorliegen (wörtlich also: „Vom König Geliebte/ Lieblinge des Königs sind, die sprechen werden", etc.). Dass der Fokus tatsächlich auf dem Erstglied liegt, wird durch Parallelen mit $p w$ wie die folgende bestätigt:

71. $m r r n z w p w(.) t. m . t(j)=f(j)\ulcorner q r j z p n n \underline{d} . t=(j) m$ ${ }^{c} b=f$ (Edel 1953: 328 rechts)

„Wer in dieses Grab meiner Stiftung nicht in unreinem Zustand eintreten wird, (Fokus:) den liebt der König (....)“

Nun gibt es auch Fälle mit aktivischem $m r r$, die einer entsprechenden Deutung wohl inhaltliche Schwierigkeiten entgegensetzen:

72. $m r r h z z$ sw $n t r$ C $3 b 3 b \underline{d} w \underline{d d} . t=f$ (folgt Opferformel) (CG 20523 = Lange \& Schäfer 1902, II: 123; ähnlich CG 20046 = ebd. I: 55) 
Dies muss sinngemäß etwa bedeuten: „Wer will, dass der große Gott, der Herr von Abydos, ihn lobt, der soll sprechen (...)“. Auch hier wird $\underline{d} d . t=f$ traditionell als $s \underline{d} . t j=f j$ aufgefasst, aber eindeutig so geschriebene Belege kenne ich nicht. Ich vermute, dass in Fällen wie diesem vielmehr die oben behandelte Konstruktion mit Objektfokus vorliegt und die Phrase mrr... $3 b d w$ als Topic dem Satz vorangestellt ist:

„Wer will, dass der große Gott, der Herr von Abydos, ihn lobt - er soll sprechen (Fokus:) (Opferformel).“

\section{Objektfokus unmarkiert}

Auch wo funktional eine Fokussierung vorliegt, muss diese im Ägyptischen nicht immer grammatisch markiert sein. So ist insbesondere bekannt, dass nicht jede fokussierte Adverbialie durch ein Zweites Tempus eingeleitet wird (Cassonet 2000: 57-60 gibt an, dass der Gebrauch des Zweiten Tempus erst von der Ramessidenzeit an obligatorisch wird). Es soll hier nicht versucht werden, genauere Regeln dafür zu finden, wann ein inhaltlicher Fokus grammatisch markiert wird und wann nicht. Das Folgende sind einige Beispiele für fokussierte direkte Objekte, insbesondere Frageworte, die nicht auf eine der bisher beschriebenen Weisen markiert sind:

73. $j r y=j m$ (Admonitions 2, $9=$ Helck 1995: 9) "Was soll ich tun?"

74. jni.n= $k$ jusst hnt $=s$ (CT VII $230 \mathrm{~h})$ „Was hast du darin mitgebracht?"

75. $j w=\underline{t}(r) d i . t n=j j h \underline{d} 3 y=(j) \underline{t} w r p 3 j w h r j-j b \ldots j w=j$ (r) dit.t $n=k$ t3y wh3.t (Horus \& Seth 5,12f. = LES 43)

„Was willst du mir geben, damit ich dich zur Mitteninsel übersetze?“ - „Ich gebe dir diesen Kuchen"

76. $j r y=j j h r=t m$ bt 3 (pLeiden $371,1=$ Gardiner \& Sethe 1928: Tf. 7; 19. Dyn.)

„Was habe ich dir Unrechtes getan?"

Noch etwas anders gelagert sind Fälle, in denen zwar ein objektbildendes Fragewort nicht selbst als Fokus markiert wird, dafür aber die das Fragewort umgebende Phrase als ganze:

77. jy. $n=k j r=[f] r$ wnm jšst (CT III $50 \mathrm{~d}$ ) „Um was zu essen, bist du gekommen?"
Inhaltlich fokussiert ist eigentlich nur das Fragewort jšst, aber der Fokus wird auf die gesamte Phrase $r$ wnm jšst expandiert. In grammatischer Hinsicht liegt damit nicht mehr ein Objektfokus, sondern ein Adverbialfokus vor, und es erscheint infolgedessen das Zweite Tempus $j y . n=k$. Ebenso:

78. $m r r \underline{t} w j t j=k j r i=k n=f j \check{s} s t(C T$ V $122 \mathrm{~b}-\mathrm{d})$

„Weil du was für ihn tust, liebt dein Vater dich?“

\section{Literatur}

J. P. Allen 2002: The Heqanakht Papyri, New York.

W. Barta 1969: Das Gespräch eines Mannes mit seinem Ba (Papyrus Berlin 3024), Berlin.

A. M. Blackman 1932: Middle Egyptian Stories, Bruxelles.

A. M. Blackman 1988: The Story of King Kheops and the Magicians Transcribed from Papyrus Westcar, Reading.

J. F. Borghouts 1986: Prominence Constructions and Pragmatic Functions, in G. Englund \& P. J. Frandsen, Crossroad, København, 45-70.

P. Cassonet 2000: Les temps seconds i-sdm.f et i-iri.f $s d m$ entre syntaxe et sémantique, Paris.

E. W. Castle 1993: The Dedication Formula ir.n.fm mnw.f, Journal of Egyptian Archaeology 79: 99120.

J. Černý \& A. H. Gardiner 1957: Hieratic Ostraca I, Oxford.

CT $=$ A. de Buck 1935ff.: The Egyptian Coffin Texts, 7 Bde., Chicago.

L. Depuydt 1994: On a Late Egyptian and Demotic Idiom, Revue d'Égyptologie 45: 49-73.

É. Doret 1991: Cleft-sentence, substitutions et contraintes sémantiques en égyptien de la première phase, Lingua Aegyptia 1: 57-96.

E. Edel 1953: Inschriften des Alten Reiches, Mitteilungen des Instituts für Orientforschung 1: 327-336.

E. Edel 1955/64: Altägyptische Grammatik, 2 Bde. Roma.

M. El-Khadragy 2002: The Edfu Offering Niche of Qar in the Cairo Museum, Studien zur Altägyptischen Kultur 30: 203-228.

R. O. Faulkner 1969: The Ancient Egyptian Pyramid Texts. Supplement of Hieroglyphic Texts, Oxford.

H.-W. Fischer-Elfert 2000: Rezension zu J. F. Quack, Studien zur Lehre für Merikare, Lingua Aegyptia 7: 261-267.

M.-P. Foissy-Aufrère \& S. Aufrère \& Chr. Loury 1985: Egypte \& Provence. Civilisation, survivances et „cabinets de curiosités“, Avignon. 
A. H. Gardiner 1909: Die Erzählung des Sinuhe und die Hirtengeschichte (HP 5), Leipzig.

A. H. Gardiner 1957: Egyptian Grammar being an Introduction to the Study of Hieroglyphs, 3rd ed., London.

A. H. Gardiner \& T. E. Peet 1952/5: The Inscriptions of Sinai. Second Edition Revised and Augmented by Jaroslav Černý, 2 Bde., London.

A. H. Gardiner \& K. Sethe 1928: Egyptian Letters to the Dead, London.

M. Gilula 1976: An Unusual Nominal Pattern in Middle Egyptian, Journal of Egyptian Archaeology 62: 160-175.

H. Grapow 1958: Die Medizinischen Texte in hieroglyphischer Umschreibung autographiert, Berlin.

S. I. Groll 1967: Non-Verbal Sentence Patterns in Late Egyptian, London.

I. Hafemann 2002: Zum Zusammenspiel von Semantik und Syntax ägyptischer Verben, Lingua Aegyptia 10: 151-210.

W. Helck 1995: Die „Admonitions“. Pap. Leiden I 344 recto, Wiesbaden.

R. S. Jackendoff 1972: Semantic Interpretation in Generative Grammar, Cambridge.

F. Junge 1981: Nominalsatz und Cleft Sentence im Ägyptischen, in D. W. Young (Hrsg.), Studies presented to Hans Jakob Polotsky, Beacon Hill, 431-462.

L. Klebs 1922: Die Reliefs und Malereien des Mittleren Reiches, Heidelberg.

R. Koch 1990: Die Erzählung des Sinuhe, Bruxelles. KRI $=$ K. A. Kitchen 1975ff.: Ramesside Inscriptions, 8 Bde., Oxford.

P. Lacau 1949: Une stèle juridique de Karnak, Le Caire.

P. Lacau 1961: La stèle J. E. 59636 du Musée du Caire, in Mélanges Mariette (BdE 32), Le Caire, 211-227.

H. O. Lange \& H. Schäfer 1902: Grab- und Denksteine des Mittleren Reichs im Museum von Kairo, Nr. 20001-20780, 4 Teile, Berlin.

C. Leitz 1994: Tagewählerei. Das Buch ḩ3t nhhh ph.wy $d t$ und verwandte Texte, 2 Bde., Wiesbaden.

LEM $=$ A. H. Gardiner 1937: Late-Egyptian Miscellanies, Bruxelles.

LES = A. H. Gardiner 1932: Late Egyptian Stories, Bruxelles.

A. Loprieno 1995: Ancient Egyptian. A Linguistic Introduction, Cambridge.

LRL = J. Černý 1939: Late Ramesside Letters, Bruxelles.

P. Montet 1930-35: Les tombeaux de Siout et de Deir Rifeh II, Kêmi 3: 45-111.

E. Naville 1886: Das aegyptische Todtenbuch der XVIII. bis XX. Dynastie, 2 Bde., Berlin.

F. Neveu 1994: Vraie et pseudo cleft sentence en néo-égyptien, Lingua Aegyptia 4: 191-212.
R. B. Parkinson 1991: The Tale of the Eloquent Peasant, Oxford.

T. E. Peet 1923: The Rhind Mathematical Papyrus, London.

C. Peust 2004: Das Ägyptische als afrikanische Sprache, in Th. Schneider (Hrsg.), Das Ägyptische und die Sprachen Vorderasiens, Nordafrikas und der Ägäis, Münster, 321-407.

C. Peust 2006: Die Konjugation der Verben $r h$ „wissen" und hm „nicht wissen" im Älteren Ägyptisch, Studien zur Altägyptischen Kultur 35: 219-243.

A. Piank off 1944: Le Livre des Quererts, Bulletin de l'Institut Français d'Archéologie Orientale 42: $1-62$.

H. J. Polotsky 1962: Nominalsatz und Cleft Sentence im Koptischen, Orientalia 31: 413-430.

PT $=$ K. Sethe 1908ff.: Die altägyptischen Pyramidentexte nach den Papierabdrücken und Photographien des Berliner Museums, 4 Bde., Leipzig.

J. E. Quibell et al. 1898: The Ramesseum and the Tomb of Ptah-hetep, London.

D. Randall-Maciver \& C. L. Woolley 1911: Buhen, Philadelphia.

C. Reintges 1998: Mapping Information Structure to Syntactic Structure: One Syntax for $j n$, Revue d'Égyptologie 49: 195-220.

H. Satzinger 1997: Gott gibt dem König Leben, Zeitschrift für Ägyptische Sprache und Altertumskunde 124: 142-156.

W. Schenkel 2005: Tübinger Einführung in die klassisch-ägyptische Sprache und Schrift, Version blau, Tübingen.

K. Sethe 1916: Der Nominalsatz im Ägyptischen und Koptischen, Leipzig.

K. Sethe 1928: Aegyptische Lesestücke zum Gebrauch im akademischen Unterricht, 2. Aufl., Leipzig.

D. P. Silverman 1980: An Emphasized Direct Object of a Nominal Verb in Middle Egyptian, Orientalia 49: 199-203.

P. Tresson 1928: Une petite stèle inédite du moyen empire, Kêmi 1: 69-82.

Urk I = K. Sethe 1933: Urkunden des Alten Reichs, 2. Aufl., Leipzig.

Urk IV $=$ W. Helck \& K. Sethe $1927 \mathrm{ff} .:$ Urkunden der 18. Dynastie, Berlin/Leipzig.

A. Varille 1954: La stèle du mystique Béky, Bulletin de l'Institut Français d'Archéologie Orientale 54: 129-135.

P. Vernus 1976: Deux inscriptions de la XII ${ }^{\text {e dynas- }}$ tie provenant de Saqqara, Revue d'Égyptologie 28: 119-138.

P. Vernus 1987: Études de philologie et de linguistique (VI), Revue d'Égyptologie 38: 163-181

P. Vernus 1991: Le rhème marqué: typologie des emplois et effets de sens en Moyen Egyptien, Lingua Aegyptia 1: 333-355. 
P. Vernus 2006: Pronoms interrogatifs en égyptien de la première phase, Lingua Aegyptia 14: 145178.

W. Westendorf 1962: Grammatik der Medizinischen Texte, Berlin.

Z. Žába 1956: Les maximes de Ptaḥhotep, Prague.

J. Zeidler 1999: Pfortenbuchstudien, 2 Bde., Wiesbaden.

\section{Summary}

The Egyptian language possesses well-known constructions for focalizing a subject (subjectfocalizing cleft sentence $j n+$ subject + active participle) as well as an adverbial phrase (second tense). It is argued that there is, in addition, a specialized con- struction for marking a focussed direct object in Old and Middle Egyptian, namely relative form (or passive participle) + object, which is obligatorily inverted to object + relative form when the focussed element is an interrogative pronoun. The term object-focalizing cleft sentence is proposed for these constructions. The object-focalizing cleft sentence cannot synchronically be analyzed as a regular nominal sentence because the bipartite nominal sentence is no longer productive in Middle Egyptian.

An alternative means of focussing a logical direct object is to transform it into a prepositional phrase and to make use of the second tense construction. This possibility, which is available up to the Late Egyptian period, has been described in the literature but is still underestimated in its importance; additional examples are forwarded in this paper. 